\title{
Identification of Indoor Contaminant Source by Inverse Zonal Method
}

\author{
Aziz Azimi, Ehsan Daneshgar \\ Mechanical Engineering Department, Shahid Chamran University of Ahvaz \\ Golestan Ave., Ahvaz, Iran \\ a.azimi@scu.ac.ir; ehsan.daneshgar@gmail.com
}

\begin{abstract}
Traditionally the indoor contaminant distribution can be obtained by CFD methods. However, it is too time consuming and costly. This paper reports the development of a zonal approach as a simple model to provide an alternative and inexpensive approach for indoor contaminant distribution. Experimental measurements and CFD results are employed to validate the reliability of this method for direct problem. This comparison shows the capability of zonal method for solution of air flow in indoor environments. Finally, inverse zonal method is developed to predict the unknown indoor contaminant source location and strength (emission rate) knowing source release time. The inverse analysis for solving the identification problem is Levenberg-Marquardt method. The results show that this inverse analysis can identify the characteristics of a gaseous emission source using a zonal model as the direct problem.
\end{abstract}

Keywords: Zonal Method, Air Distribution, Contaminant Distribution, Inverse Analysis, Levenberg-Marquardt Method.

\section{Nomenclatures}

\section{Symbol Meaning}

$A_{i, j}$

Cross section area between zones $\mathrm{i}$ and $\mathrm{j}\left[\mathrm{m}^{2}\right]$

$C_{d}$

g Gravity acceleration $\left[\mathrm{m} / \mathrm{s}^{2}\right]$

I Total numbers of measurement values

J The sensitivity matrix

$p \quad$ Pressure [pa]

$S_{c} \quad$ The source of pollution

$S(\boldsymbol{P}) \quad$ Target function for minimization

$u_{i} \quad$ Component of velocity vector $[\mathrm{m} / \mathrm{s}]$

$V_{i-j} \quad$ Air velocity from zone $\mathrm{j}$ to zone $\mathrm{i}[\mathrm{m} / \mathrm{s}]$

$\boldsymbol{Y} \quad$ Vector of the measured concentration values

\section{Symbol Meaning}

C

$D$

$h$

$\boldsymbol{I}$

$\dot{\mathrm{m}}_{i, j}$

$\boldsymbol{P}$

$S_{i}$

$\mathrm{t}$

$V_{i}$

$x_{i}$

$\mu$

$\rho$

Damping parameter

Density of air $\left[\mathrm{kg} / \mathrm{m}^{3}\right]$

\section{$\underline{\text { Subscripts }}$}

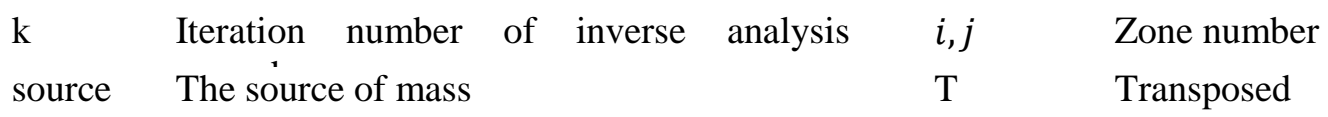




\section{Introduction}

People spend more than $80 \%$ of their life time in indoor environments [1]. The level of protection of a building against the intentional or accidental release of chemical agents is crucial. Both scenarios could endanger life and safety of the building occupants. Quickly identifying the characteristics (e.g., location and strength) of contaminant source in short time is important for taking prompt response measures to protect occupants and mitigate losses.

Todays, the inverse analyses, especially during the last three decades, relying on the accuracy of measurement data for analysing mass and heat transfer systems have been more attention. In this way, inverse methods are used in various practical problems in fields of science and engineering, especially in the design and control of fluid, thermal and pollutant systems. The most application of inverse methods is to determine the boundary conditions, the position and strength of heat source/sink, the position and rate of pollution emission and gases release in the environment and to estimate the unknown parameters such as thermophysical properties of the material.

In the other words, in the inverse pollution problems involving gaseous pollution sources, the transient effects associated with pollutant diffusion or convection are important. In fact, the pollution entering into space or generated in space is estimated using the transient concentration distribution at certain points near the boundaries of geometric. Thus, the identification of a contaminant source is an inverse problem compared to the prediction of contaminant dispersion. Although much work has been conducted on the inverse problem in heat transfer, ground water transport and atmospheric constituent transport, only a little work has been published on the determination of indoor contaminant source [2].

Zhang and Chen [3] applied inverse CFD modelling to locate the source of a contaminant inside an enclosed space. They used quasi-reversibility method which solves the specie mass conservation equation with reversed time step. They applied this method to find the source of a contaminant in an aircraft cabin and an office building. Liu and Zhai [4] proposed a probability-based CFD modelling method for identifying the location of an instantaneous source. The application of this model was tested for a 2-D office and 3-D aircraft cabin. The inverse modelling was conducted with a known contaminant release time. The model showed an acceptable accuracy in predicting the contaminant source location.

Running a CFD program cannot give a fast real-time result; hence, Liu and Zhai [5] developed a probability-based inverse multi-zone method for identifying source location in a building with many components. The method requires the knowledge of exact source activation time. Bastani et al. [6] developed a method which uses artificial neural network as a statistical analysis integrated with a multi-zone airborne contaminant transport model, CONTAM. This method was able to predict the source location in more than $90 \%$ of the cases when the building was monitored by three or more sensors.

Previously inverse analysis was applied to multi-zone and CFD methods. But due to the limitation of the multi-zone method for large environments and the time consuming CFD methods, in this paper, a faster inverse zonal method is developed. Inverse zonal method in comparison to multi-zone method has more accurate results and is much faster than CFD methods. In fact, the goal and novelty of this research is the identification of unknown parameters using an inverse simplified CFD-type model with a good accuracy in less time consumption and computation cost.

In this manner, a zonal approach as the direct problem is developed to provide an alternative and inexpensive approach for the indoor contaminant distribution. For validating the solution of the direct problem, its results are compared to experimental data and CFD results. This comparison shows the capability of zonal method to obtain indoor air flow/contaminant distribution within engineering accuracy. Finally, an inverse zonal model is developed to predict the unknown indoor contaminant source location and strength knowing source release time. The inverse method is LevenbergMarquardt method. The results show the powerful ability of this inverse method to identify the characteristics of a gaseous emission source using a zonal model as the direct problem.

\section{Direct Problem: Zonal Method}

To obtain indoor contaminant air distribution, the Inard Pressure-Zonal [7] method is used as the flow simulation model for the ventilated rooms. In this way, the space is subdivided into a structured Cartesian grid of control volumes or cells. It provides air volume of each zone, airflow rate between zones, and wall areas of the zone adjacent to the walls for indoor environment simulation. The fundamental assumptions of the model are [8]:

1. Each zone is well mixed and all properties are uniform inside it;

2. The air density is constant;

3. The air and species in the air are transported from zone to zone only by the convection, and the diffusion is neglected; 
4. The contaminant is released when the indoor flow field reaches to steady state;

Based on the above assumptions, the mass balance for each zone (zone i) can be expressed as the following equation (Fig. 1):

$$
\sum_{j=1}^{6} \dot{m}_{i, j}+\dot{m}_{i, \text { source }}=0
$$

For vertical faces with horizontal flow, the air flow rate between zones can be written as:

$$
\begin{gathered}
\dot{m}_{i, j}=\varepsilon_{i, j} \sqrt{2 \rho_{i}} \times C_{d} \times A_{i, j}\left|p_{i}-p_{j}\right|^{0.5} \\
\varepsilon_{i, j}=\operatorname{sign}\left(p_{i}-p_{j}\right)
\end{gathered}
$$

For horizontal faces with vertical flow, the pressure term must account for the hydrostatic pressure difference of the air [7]:

$$
\begin{gathered}
\dot{m}_{i, j}=\varepsilon_{i, j} \sqrt{2 \rho_{i}} \times C_{d} \times A_{i, j}\left|p_{i}-p_{j}+0.5\left(\rho_{i} g h_{i}+\rho_{j} g h_{j}\right)\right|^{0.5} \\
\varepsilon_{i, j}=\operatorname{sign}\left(p_{i}-p_{j}+0.5\left(\rho_{i} g h_{i}+\rho_{j} g h_{j}\right)\right)
\end{gathered}
$$

As can be seen, all sentences in the equation of momentum conservation are summarized in a coefficient and this equation has been simplified. Comparison of the results of zonal method with the experimental data shows that for most flows of convective heat transfer, this coefficient can be had a value between 0.6 and 0.8 , and it produces good results [911]. In the air zonal model, the ideal gas equation is used to calculate the density.

The conservation equation of gaseous pollution in air can be as the following partial differential equation [6]:
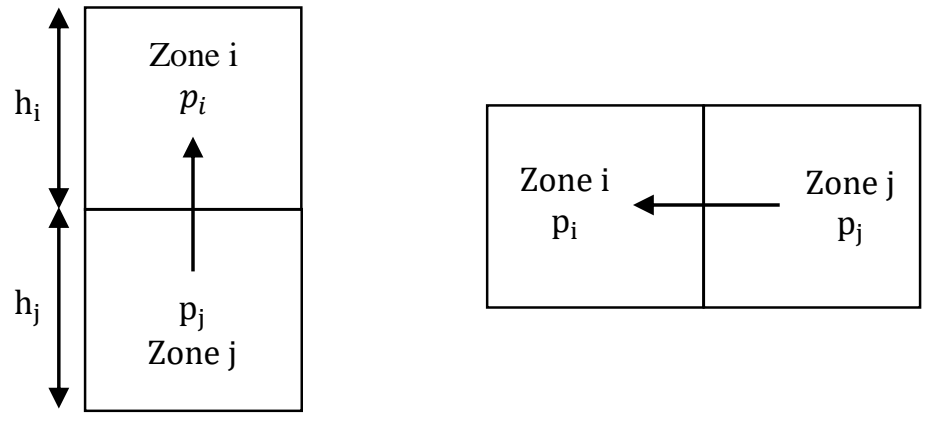

Fig. 1: Flow between two zones with interface.

$$
\frac{\partial C}{\partial t}=-\frac{\partial}{\partial x_{i}}\left[u_{i} C\right]+\frac{\partial}{\partial x_{i}}\left[D \frac{\partial C}{\partial x_{i}}\right]+S_{C} \quad \text { where } \quad i=1,2,3 \text { (for three dimensional problems) }
$$

The left hand side of Eq. (4) is the rate of concentration changes with time. The terms at the right hand side of the equation are the convective, diffusion and source of pollution in the environment. In order to model the propagation of air and pollution by zonal method, as before written, it is assumed that each volume is thoroughly mixed and all properties are uniform. The diffusion phenomenon of pollution is neglected and it is assumed that air pollution can be transported from one zone to adjacent zone only by convective phenomenon. Based on the above assumptions, the mass balance equation of pollution for each zone is [6]:

$$
V_{i} \frac{d C_{i}}{d t}=\sum_{j \neq i} V_{j-i} C_{i}-\sum_{j \neq i} V_{i-j} C_{i}+\sum S_{i}
$$




\section{Inverse Problem: Levenberg-Marquardt Method}

Identifying the contaminant source location and strength by sensors' data is known as an inverse problem. Finding source of the contaminant released into indoor can be useful, because at this time, appropriate actions can be taken to deal with this event. The released contaminant in enclosed environment can be in gas, liquid, or solid phase. This paper concerns gaseous contaminant transport. Generally, when a contaminant releases inside a building, the contaminant concentration in a sensor location is a function of the source location, source release time and source strength. Here, Levenberg-Marquardt method is used to predict the contaminant source location and strength in a mechanically ventilated indoor environment knowing source release time.

The solution of the inverse problem for the estimation of unknown parameters is based on the minimization of the ordinary least squares norm:

$$
S(\mathbf{p})=\sum_{l=1}^{I}\left[Y_{l}-C_{l}(\mathbf{p})\right]^{2}
$$

where the estimated concentrations $C_{l}(\mathbf{p})$ are obtained from the solution of the direct problem at the measurement location and $Y_{l}$ are the measured concentrations. To minimize the least squares norm given by Eq. (6), derivatives of $S$ (p) with respect to each of unknown parameters must be equal to zero. For the nonlinear inverse problems, a Taylor series expansion around $\mathbf{p}^{k}$ is used to linearize the vector of estimated concentrations, $\mathbf{C}(\mathbf{p})$. Finally, the following equation can be used to estimate the unknown parameters in an iterative manner:

$$
\mathbf{p}^{k+1}=\mathbf{p}^{k}+\left[\left(\mathbf{J}^{k}\right)^{T} \mathbf{J}^{k}\right]^{-1}\left(\mathbf{J}^{k}\right)^{T}\left[\mathbf{Y}-\mathbf{C}\left(\mathbf{p}^{k}\right)\right]
$$

where the sensitivity or Jacobian matrix is written as:

$$
\mathbf{J}(\mathbf{p})=\left[\frac{\partial \mathbf{C}(\mathbf{p})}{\partial \mathbf{p}}\right]
$$

The iterative procedure given by Eq. (7) requires the matrix $\left(\mathrm{J}^{\mathrm{k}}\right)^{\mathrm{T}} \mathrm{J}^{\mathrm{k}}$ to be non-singular. For ill-conditioned problems, $\left|\left(\mathrm{J}^{\mathrm{k}}\right)^{\mathrm{T}} \mathrm{J}^{\mathrm{k}}\right| \approx 0$, the so called Levenberg-Marquardt Method alleviates such difficulties by utilizing a damping matrix in an iterative procedure. To derive this technique, Levenberg [12] first, based on his observations, combined the steepest decent and Gauss-Newton methods:

$$
\mathbf{p}^{k+1}=\mathbf{p}^{k}+\left[\nabla^{2} S(\mathbf{p})+\boldsymbol{\mu}^{k} \mathbf{I}\right]^{-1}\left(\mathbf{J}^{k}\right)^{T}\left[\mathbf{Y}-\mathbf{C}\left(\mathbf{p}^{k}\right)\right]
$$

If $\mu$ becomes very large, Eq. 10 tends to the steepest decent method (the linear approximation by starting from the initial value), if $\mu$ becomes very small, Eq. 9 tends to the Gauss-Newton method (the quadratic approximation by approaching to the minimum value). By assuming a linear approximation of errors or a small residual of errors, Eq. (9) can be rewritten as follows [13-14]:

$$
\mathbf{p}^{k+1}=\mathbf{p}^{k}+\left[\left(\mathbf{J}^{k}\right)^{T} \mathbf{J}^{k}+\boldsymbol{\mu}^{k} \mathbf{I}\right]^{-1}\left(\mathbf{J}^{k}\right)^{T}\left[\mathbf{Y}-\mathbf{C}\left(\mathbf{p}^{k}\right)\right]
$$

After that, Marquardt [15] suggested to have much smaller gradient in the minimization direction together with estimating local curvature information, it is better to be used the diagonal matrix of Hessian matrix instead of the identity matrix in Eq. (10):

$$
\mathbf{p}^{k+1}=\mathbf{p}^{k}+\left[\left(\mathbf{J}^{k}\right)^{T} \mathbf{J}^{k}+\boldsymbol{\mu}^{k} \mathbf{\Omega}^{k}\right]^{-1}\left(\mathbf{J}^{k}\right)^{T}\left[\mathbf{Y}-\mathbf{C}\left(\mathbf{p}^{k}\right)\right]
$$


The purpose of the matrix term $\mu^{\mathrm{k}} \mathbf{\Omega}^{\mathrm{k}}$ is to damp oscillations and instabilities due to the ill-conditioned characteristic of the problem, by making its components large as compared to those of $\mathrm{J}^{\mathrm{T}} \mathrm{J}$, if necessary.

In fact, the insight of Marquardt was that when $\mu$ is high and we are doing essentially gradient descent, we can still get some benefit from the Hessian matrix that we estimated. This technique is quite efficient for solving linear and nonlinear least squares problems of parameter estimation in an iterative manner. On the other hand, it is one that works extremely well in practice and it is a virtual standard for optimization of medium sized nonlinear problems.

\section{Experimental Validation}

The experiment which was carried out in a test chamber and mainly focused on the contribution of contaminant in the supply air [16] is utilized for validating the present results of the direct problem. In this experiment, a supply air inlet $(0.2 \mathrm{~m}$ $\times 0.2 \mathrm{~m})$ and an exhaust air outlet $(0.3 \mathrm{~m} \times 0.18 \mathrm{~m})$ are arranged in two side walls (Fig. 2). There are no inner heat sources and contaminant sources in the chamber. The wall boundary is treated as adiabatic. The air change rate of the chamber is 7.4 $\mathrm{ACH} . \mathrm{CO} 2$ was used as the tracer gas in this experiment and it was released in the supply air duct close to inlet. The emission rate is kept at $25 \mathrm{~L} / \mathrm{min}$ by a flow rate controller in order to create a constant supply concentration. Three sensors were placed in the room space to record the transient concentration (Fig. 3).

The further transient concentrations at the three sensor locations have been calculated using zonal method with 175 zones. The comparison between the calculated results and experiment measurements are shown in Fig. 4. Because there inevitably exist some recording errors and delay of $\mathrm{CO} 2$ detectors at the beginning period of emission, the relative errors between the measured and calculated values are a little higher. From the results, it can be concluded that the proposed expression to solve the direct problem has an acceptable accuracy in determining the transient concentration distribution.

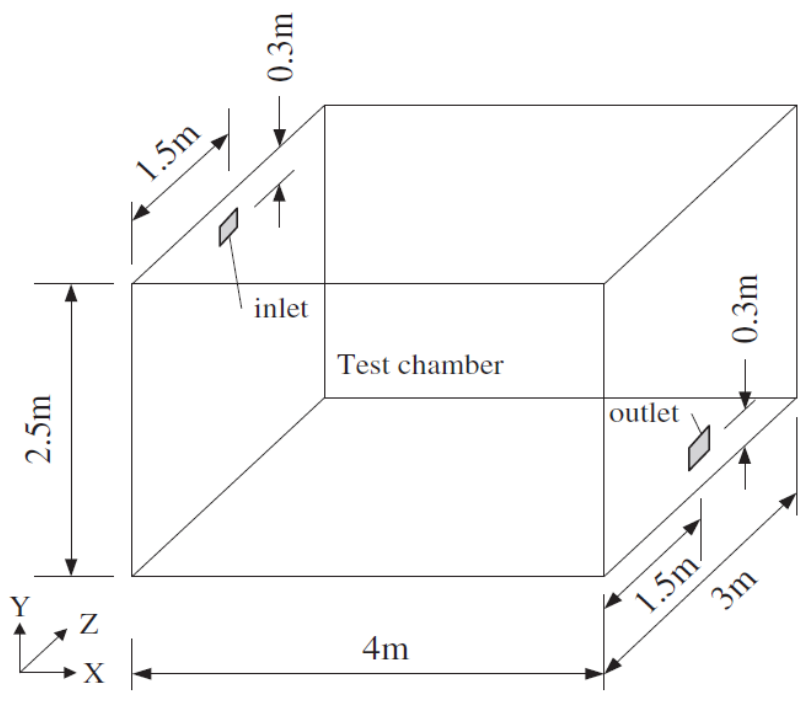

Fig. 2. Sketch map of chamber [1].

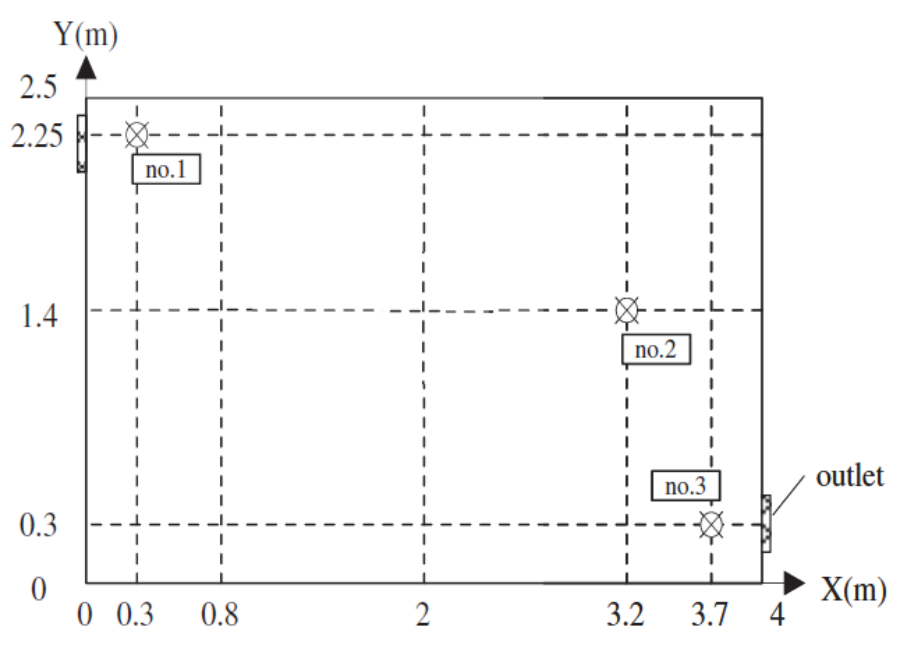

Fig. 3. Location of three sensors [1].

\section{The Results of the Inverse Problem}

According to the previous validation, by considering a room space and its indoor contaminant source, the unknown characteristics of the contaminant source including the source strength and location are estimated using inverse analysis. The dimension of room and the supply and exhaust air locations are shown in Fig. 5. The supply air velocity is considered 0.5 $\mathrm{m} / \mathrm{s}$ and is not contaminated. The only cause of indoor production and emission of contaminant is the gaseous contaminant source. Layout of contaminant source and contaminant concentration sensors is shown in Fig. 6.

Later on, three issues including estimation of the constant contaminant source strength by knowing its location, time varying contaminant source strength by knowing its location and estimation of constant contaminant strength without knowing its location have been studied by using the inverse zonal method. For each issue, the sensitivity and inverse analyses have been done. For simulating numerically the real data of a sensor, the calculated contaminant concentration using the direct problem is used as follows: 


$$
Y_{l}=C_{l} \pm \alpha \times \max \left(C_{l}\right) \quad l=1,2,3, \cdots, I
$$

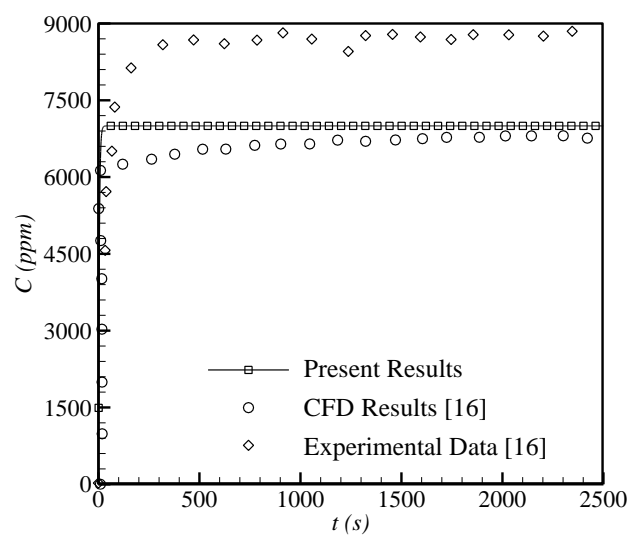

(a) Sensor no. 1

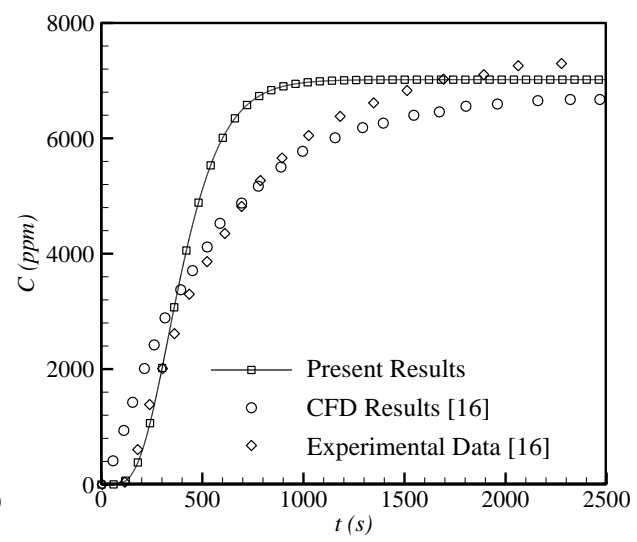

(b) Sensor no. 2.

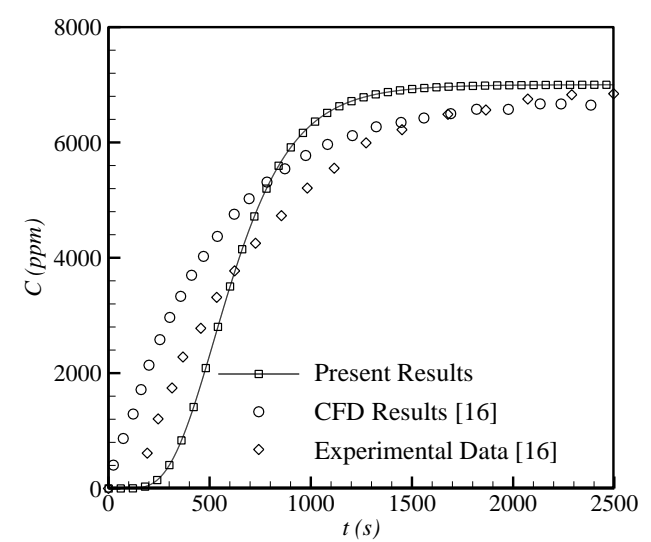

(c) Sensor no. 3

Fig. 4: Comparison of experimental, CFD and zonal results.

\subsection{Constant Contaminant Source}

In this section, the strength of a contaminant source is considered constant and equal to $5 \mathrm{mg} / \mathrm{s}$. Then, the sensitivity analysis is done and shown in Fig. 7-(a) for this inverse problem. By assuming different noisy values for the contaminant sensor, the contaminant source strength is estimated by Levenberg-Marquardt method and is shown in Table 1. The initial guess for contaminant source strength is $2 \mathrm{mg} / \mathrm{s}$.

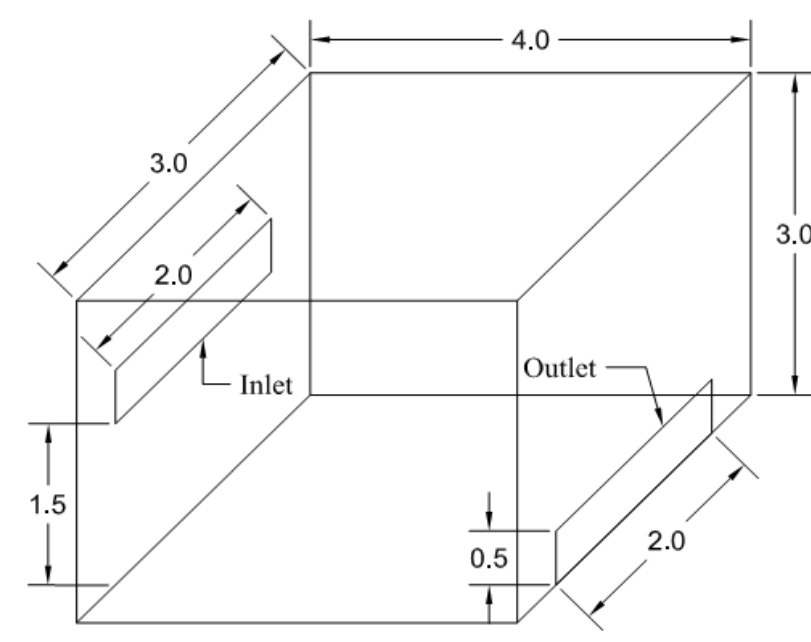

Fig. 5: Room dimension and the supply/exhaust air location.

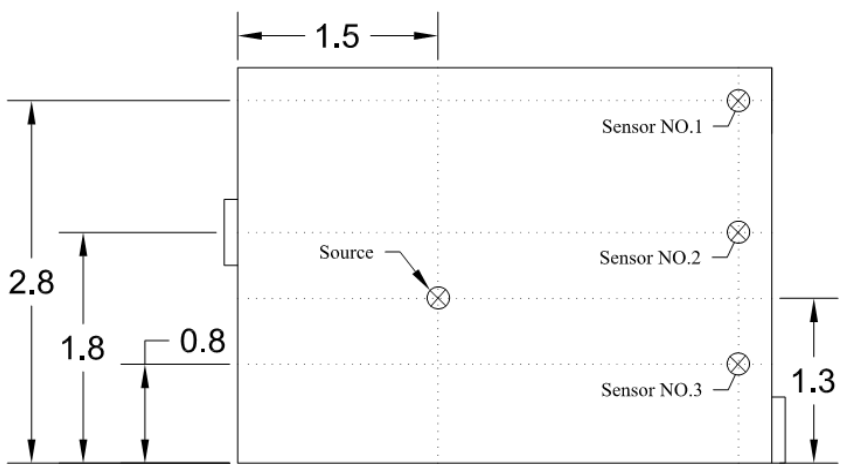

Fig. 6: Contaminant source and sensors layout.

\subsection{Time Variable Contaminant Source}

The inverse zonal method is used to estimate the time varying contaminant source strength according to the two unknown parameters $\mathrm{A}$ and $\mathrm{B}$ in the form of $\mathrm{A}+\mathrm{B} \mathrm{t}$. For this inverse problem, it is assumed that location of contaminant source and released time are known, but the source strength is unknown. The values of $\mathrm{A}$ and $\mathrm{B}$ are $7 \mathrm{mg} / \mathrm{s} \mathrm{and} 2 \mathrm{mg} / \mathrm{s} 2$, respectively. The initial guess for both unknown parameters ( $\mathrm{A}$ and $\mathrm{B}$ ) is considered equal to one.

Sensitivity analysis has been used for these unknown parameters and is shown in Fig 7-(b). As can be seen, the sensitivity of parameter B to the sensor data is more than that of parameter A over the time and this difference increases excessively by passing of time. It means that, to estimate parameters A and B, the estimation of parameter B is much faster and more accurate because of its high sensitivity. By assuming various noisy values for the contaminant sensor, two unknown parameters of contaminant source strength have been calculated and shown in Table 2, using Levenberg-Marquardt method. 


\subsection{Constant Contaminant Source and Unknown Location}

By assuming an unknown constant contaminant source strength and location along the length of the room, they are estimated using the inverse zonal method. The contaminant source strength is considered $7 \mathrm{mg} / \mathrm{s}$. Notice that in this case, the location is considered as a zone number along the length of the room. Therefore, due to the creation of eight zones along the length of the room, the contaminant and sensor locations are in zone numbers two and eight, respectively. The initial guess for the contaminant source strength in the room is equal to $3 \mathrm{mg} / \mathrm{s}$ and for the contaminant location along the length of the room is considered zone number one. Sensitivity analysis for source strength and location is shown in Fig 7-(c).

According to the performed sensitivity analysis, the sensitivity of contaminant source location is increased over the time and this means the accurate estimation of source location is related to the source strength. The results of inverse analysis are shown in Table 3. As shown, Levenberg-Marquardt method is able to calculate the strength and location of contaminant source with acceptable error.

\section{Conclusion}

The aim of this research is to develop a method involving sensors to have the ability of estimating the characteristics of contaminant source in closed environments. In such methods, the speed of recognizing unknown parameters in order to the safety of residents against intentional or accidental release of contaminant is of the most importance. In this paper, the inverse zonal method for identification of indoor contaminant source is developed.

For this purpose, simulation of contaminant emission into the indoor environment as the direct problem has been performed by the zonal method. In this problem, the contaminant has been entered the room through air conditioning and there have been no inner heat sources and contaminant sources in the room. The direct problem has been validated by comparing its results to experimental data and CFD results. According to this comparison, the direct problem yields results within engineering accuracy. After validation of the zonal method, a new sample space has been defined in order to investigate the application of inverse zonal method in calculating the strength and location of unknown contaminant sources.

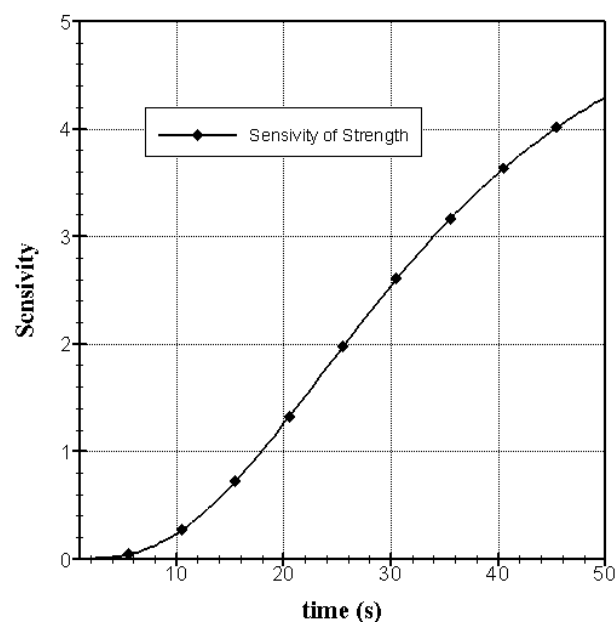

(a) Constant source strength

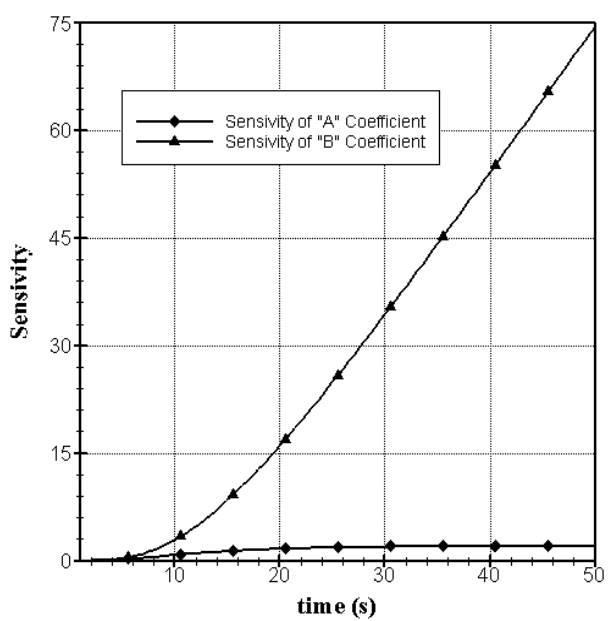

(b) Time varying source strength Fig. 7: Sensitivity analyses.

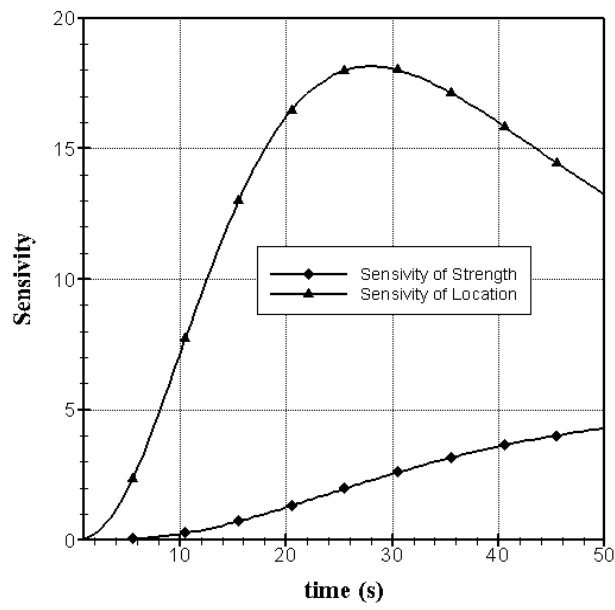

(c) Constant source strength and location

Table 1: Estimation of constant contaminant source strength.

\begin{tabular}{|c|c|c|c|}
\hline Sensor Noise & Est. Strength & Iter. No. & Est. Strength Error \% \\
\hline 0 & 5.00 & 4 & 0 \\
\hline 0.001 & 5.00 & 4 & 0 \\
\hline 0.01 & 5.05 & 4 & 1.00 \\
\hline 0.05 & 5.29 & 4 & 5.80 \\
\hline 0.1 & 5.59 & 4 & 11.80 \\
\hline 0.2 & 6.19 & 4 & 23.80 \\
\hline
\end{tabular}


Table 2: Estimation of time varying contaminant source strength.

\begin{tabular}{|c|c|c|c|c|c|}
\hline Sensor Noise & Iter. No. & Par. A & Par. A Error \% & Par. B & Par. B Error \% \\
\hline 0 & 3 & 7.00 & 0 & 2.00 & 0 \\
\hline 0.001 & 3 & 7.22 & 3.14 & 1.99 & 0.50 \\
\hline 0.01 & 3 & 9.22 & 31.71 & 1.99 & 0.50 \\
\hline 0.05 & 3 & 18.10 & 158.57 & 1.96 & 2.00 \\
\hline 0.1 & 3 & 29.20 & 317.14 & 1.92 & 4.00 \\
\hline 0.2 & 3 & 51.41 & 634.42 & 1.85 & 7.50 \\
\hline
\end{tabular}

Table 3: Estimation of constant contaminant source strength and location.

\begin{tabular}{|c|c|c|c|c|}
\hline Sensor Noise & Iter. No. & Est. Strength & Est. Strength Error \% & Source Loc. \\
\hline 0 & 5 & 7.00 & 0 & 2 \\
\hline 0.001 & 5 & 7.00 & 0 & 2 \\
\hline 0.01 & 5 & 7.03 & 0.42 & 2 \\
\hline 0.05 & 5 & 7.18 & 2.57 & 2 \\
\hline 0.1 & 6 & 7.36 & 5.14 & 2 \\
\hline 0.2 & 7 & 7.72 & 10.28 & 2 \\
\hline
\end{tabular}

In order to show the ability of the inverse zonal method, three sample problems have been defied by unknowing the constant contaminant source strength but its location is known, unknowing contaminant source strength which changes linearly by the time but its location is known, and unknowing the constant contaminant source strength and its location along the length of the room. Then the Levenberg-Marquardt method has been used in order to estimate the unknown parameters. This study has found that in this inverse method by taking 10 percent error in the measurement data, the unknown parameters are estimated with acceptable accuracy.

\section{References}

[1] X. Li, X. Shao, X. Ma, and Y. Lin, "An analytical expression for transient distribution of passive contaminant under steady flow field," J. Build. \& Envir., vol. 52, pp. 98-105, 2012.

[2] H. Cai, X. Li, Zh. Chen, and M. Wang, "Rapid identification of multiple constantly released contaminant source in indoor environment with unknown release time," J. Build. \& Envir., vol. 81, pp. 7-19, 2014.

[3] T. F. Zhang and Q. Chen, "Identification of contaminant sources in enclosed environments by inverse CFD modelling," J. Indoor Air, vol. 17, no. 3, pp. 167-177, 2007.

[4] X. Liu and Z. Zhai, "Location identification for indoor instantaneous point contaminant source by probability-based inverse computational fluid dynamics modelling," J. Indoor Air, vol. 18, no. 1, pp. 2-11, 2008.

[5] X. Liu, and Z. J. Zhai, "Prompt tracking of indoor airborne contaminant source location with probability-based inverse multi zone modelling," J. Build. \& Envir., vol. 44, pp. 1135-1143, 2009.

[6] A. Bastani, F. Haghighat, and J.A. Kozinski, "Contaminant source identification within a building: Toward design of immune building," J. Build. \& Envir., vol. 51, pp. 320-329, 2012.

[7] C. Inard, H. Bouia, and P. Dalicieux, "Prediction of air temperature distribution in buildings with a zonal model," $J$. Energy \& Build., vol. 24, pp. 125-132, 1996.

[8] F. Song, B. Zhao, X. Yang, Y. Jiang, V. Gopal, G. Dobbs, and M. Sahm, "A new approach on zonal modelling of indoor environment with mechanical ventilation," J. Build. \& Envir., vol. 43, pp. 278-286, 2008.

[9] E. J. Teshome and F. Haghighat, "A new generation of zonal models," J. ASHRAE Transactions, vol. 112, part 2, pp. 163-174, 2006.

[10] M. Musya, E. Wurtzb, F. Winkelmannc, and F. Allardb, "Generation of a zonal model to simulate natural convection in a room with a radiative, convective heater," J. Energy \& Build., vol. 36, pp. 589-596, 2001. 
[11] M. Musy, F. Winkelmann, E. Wurtz, and A. Sergent, "Automatically generated zonal models for building air flow simulation: principles and applications," J. Energy \& Build., vol. 37, pp. 873-881, 2002.

[12] K. Levenberg, "A method for the solution of certain non-linear problems in least squares," J. Quart. Appl. Math., vol. 2, pp. 164- 168, 1944.

[13] S. Roweis. (2016, March). Levenberg-Marquardt optimization [Online]. Available: http://www.cs.toronto.edu/ $\sim$ roweis/notes/lm.pdf.

[14] A. Ranganathan. (2016, March). The Levenberg-Marquardt Algorithm [Online]. Available: http://users-phys.au.dk/ jensjh/numeric/project/10.1.1.135.865.pdf.

[15] D. W. Marquardt, "An algorithm for least squares estimation of nonlinear parameters," J. Soc. Ind. Appl. Math., $\quad$ vol. 11, no. 2, pp. 431-441, 1963.

[16] X. Ma, X. Shao, X. Liv, and Y. Lin, “An analytical expression for transient distribution of passive contaminant under steady flow field,” J. Build. \& Envir., vol. 52, pp. 98-105, 2012. 